Part of Journal of Research of the National Bureau of Standards, Volume 25, October 1940

\title{
EFFECTS OF DRYING CONDITIONS ON PROPERTIES OF TEXTILE YARNS
}

\author{
By James G. Wiegerink ${ }^{1}$
}

\begin{abstract}
Yarns made from raw cotton, "purified" cotton, mercerized cotton, clothing wool, carpet wool, viscose ravon, cuprammonium rayon, cellulose acetate, and degummed silk were saturated with water and then exposed for periods of $1 / 2$ to 6 hours in air having temperatures of $221^{\circ}, 257^{\circ}$, and $302^{\circ} \mathrm{F}$ and absolute humidities of approximately 1,55 , and 95 percent, respectively. After conditioning the dried yarns for at least 24 hours in air of 65 -percent relative humidity and $70^{\circ} \mathrm{F}$, measurements were made of the breaking strengths, elongations at break, and moisture contents of the yarns; the fluidities of dispersions of the cellulosic fibers in cuprammonium solution, of the silk in aqueous zinc chloride, and of the acetate in aqueous acetone; and the relative affinities of the cellulosic fibers for the dye benzopurpurine $4 B$ and of the wools for acid dyes.

Heating the yarns for 6 hours at $221^{\circ} \mathrm{F}$ at each of the three humidities weakened all of them. The breaking strength and elongation at break of the viscose, cuprammonium, degummed silk, and cotton yarns decreased still more at higher temperatures. In comparison with heating at 1-percent absolute humidity, heating at 95 -percent absolute humidity resulted in lower breaking strengths and elongations at break for all yarns except the raw cotton, higher fluidities, lower affinity of cellulosic fibers, and higher affinity of wool fibers for the dyes.

Heating for $1 / 2$ hour at $302^{\circ} \mathrm{F}$ had little effect on the properties of the yarns except the viscose and cuprammonium rayon, which were adversely affected. The damage which occurred at a given temperature and humidity increased throughout the heating period.
\end{abstract}

\section{CONTENTS}

Page

I. Introduction

II. Materials

III. Procedure

IV. Results and conclusions

\section{INTRODUCTION}

Although it is common knowledge that textile fibers are degraded at elevated temperatures, the combined effects of heat and moisture have received little study. Therefore, particular attention was given to this subject in the course of the studies of textile drying carried out by the United States Institute for Textile Research and the National Bureau of Standards.

The materials which are dried in the various branches of the textile industry, including laundries and dry-cleaning plants, and the procedures used are so various that no one set of materials and conditions can be considered representative of all of them. By limiting the work to yarns made from the common fibers freed from the natural nonfibrous materials which would be removed from them in the course of

1 Research Associate at the National Bureau of Standards, representing the United States Institute for Textile Research. The establishment of this research associateship was made possible by funds contributed by 57 manufacturers. 
manufacture, and to controlled and duplicable conditions of temperature, relative humidity, airflow, and thoroughness of exposure, it was possible to obtain data which may be considered basic to all types of textile drying. The results are given in this paper.

\section{MATERIALS}

The characteristics of the yarns and the purification treatments they received are given below. Where treatment with water is indicated, distilled water was used.

The raw cotton yarn $(1 / 24)^{2}$ was washed thoroughly in water. The "purified" cotton yarn was prepared from the raw cotton yarn by successive extractions with alcohol, ether, and 1-per cent $\mathrm{NaOH}$ solution, followed by rinsing in 5-percent acetic acid solution and then in water. The mercerized cotton yarn $(2 / 25)$ was washed in a 5 -percent $\mathrm{NH}_{4} \mathrm{OH}$ solution, then rinsed in water. The clothing wool yarn (2/2.3) and the carpet wool yarn (3/1.2) were extracted with alcohol and ether, and rinsed in warm water. The viscose-rayon yarn $(1 / 30$; commercial designation 150 denier, 40 filament), the cuprammoniumrayon yarn (1/30; commercial designation 150 denier, 112 filament), and the cellulose-acetate yarn (1/30; commercial designation 150 denier, 46 filament) were washed in a warm 1-percent $\mathrm{NH}_{4} \mathrm{OH}$ solution, then rinsed in warm water. The degummed silk yarn was prepared from a raw silk yarn (4/75) by degumming with an olive oil soap solution, followed by rinsing in warm water.

\section{PROCEDURE}

The equipment for this investigation is described in detail in a previous paper. ${ }^{3}$ It provides means for controlling the temperature and humidity of air, for passing a stream of this air through a working chamber at a known rate, for supporting samples in this chamber so they are well exposed to the air stream, and for sampling the air in the working chamber and accurately determining its humidity by a gravimetric measurement of the moisture content. The temperature in the working chamber was maintained constant within $\pm 1^{\circ} \mathrm{F}$ and the humidity within \pm 1 percent throughout each test. Three humidities at each temperature were studied. Hereafter, where a low, medium, and high humidity are referred to, absolute humidities of approximately 1,55 , and 95 percent, respectively, are meant. Relative humidities corresponding to these absolute humidities are also included for comparison. ${ }^{4}$

The samples for test were soaked overnight in distilled water. The following day the excess water was removed by squeezing the yarns until their weights were approximately 50 percent more than their "dry" weights (determined by heating other samples in an oven at $220^{\circ} \mathrm{F}$ for over 12 hours). The samples were then placed in the working chamber, which was maintained at the definite temperature

\footnotetext{
2 The typp system for designating sizes of yarns is employed. The first number (1 in the case of the raw cotton yarn) indicates the number of units of which the yarn is composed, that is, the ply. The second number ( 24 in the case of the raw cotton yarn) indicates the number of thousands of yards of the finished yarn in 1 pound.

y.J.G. Wiegerink, Equipment for conditioning materials at constant humidities and at elevated temperatures, J. Research NBS 24, 639 (1940) RP1303.

"The term "relative humidity" as used in this report is the ratio of the actual pressure of water vapor to the maximum possible pressure of water vapor in the atmosphere at the same temperature, expressed as a percentage. The term "absolute humidity" as used in this report is the actual percentage by volume of water vapor in the system.
} 
and humidity of the test. The samples were kept in the chamber for different intervals of time ranging from $1 / 2$ to 6 hours. All samples came to practically constant weight before removal from the working chamber and consequently contained an amount of moisture consistent with the relative humidity and temperature of the test. These equilibrium values at the various relative humidities and temperatures are reported in a previous paper. ${ }^{5}$ It should be noted that all samples were placed in the working chamber wet, and that during evaporation of the moisture the textile was at a lower temperature than that indicated by the dry-bulb temperature in the working chamber. Thus, where a $1 / 2$ - to 1 -hour heat treatment at a certain dry-bulb temperature is indicated, the samples, although dried to equilibrium before being taken from the working chamber, may have been at the dry-bulb temperature for less than 15 minutes during the 1/2-hour exposure and less than $1 / 2$ hour during the 1-hour exposure.

The samples were removed from the working chamber and conditioned for at least 24 hours in an atmosphere of 65 -percent relative humidity at $70^{\circ} \mathrm{F}$. They were tested in this atmosphere, using a pendulum single-strand tester. The distance between the jaws of the testing machine at the start of each test was 10 inches, and the speed of separation of the jaws was 12 inches per minute. The breaking strength and elongation at break were observed.

The fluidities of dispersions in cuprammonium solution of the dried raw cotton, the purified cotton, and the viscose rayon were measured with the method described by Mease. ${ }^{6}$ These dispersions contained $0.5 \mathrm{~g}$ of the cotton, or $2.5 \mathrm{~g}$ of the viscose rayon, dry basis (calculated from the moisture content determined on a duplicate sample) for $100 \mathrm{ml}$ of dispersion. Measurements were made of the fluidity of the dried degummed silk in aqueous zinc chloride solution, density 1.67 at $70^{\circ} \mathrm{F}, 2.5 \mathrm{~g}$ of the silk, dry basis, being used for 100 $\mathrm{ml}$ of solvent. The weighed amount of the silk, cut into small pieces, was introduced into a small flask, and the required volume of zinc chloride solution added. The flask was then stoppered and placed in an oven at $113^{\circ} \mathrm{F}$ for 3 hours, with occasional swirling of the flask to ensure mixing of the contents. The solution was then cooled to $70^{\circ} \mathrm{F}$, transferred to a viscosimeter, and the viscosity measured at $70^{\circ} \mathrm{F}^{7}$

The fluidity of the dried cellulose acetate was measured in aqueous acetone, density 0.81 at $70^{\circ} \mathrm{F}, 2.5 \mathrm{~g}$ of the acetate, dry basis, being used for $100 \mathrm{ml}$ of solvent: The weighed amount of acetate was dissolved in the required volume of acetone, and kept at $70^{\circ} \mathrm{F}$ overnight. In the morning the solution was transferred to a viscosimeter and the viscosity at $70^{\circ} \mathrm{F}$ measured. The fluidities of the original samples, not dried, were measured for comparison with the dried samples.

The moisture contents of the heat-treated yarns, after conditioning them for 24 hours or more in an atmosphere of 65-percent relative humidity and $70^{\circ} \mathrm{F}$, were determined in the usual manner.

The heat-treated cellulosic yarns, namely viscose rayon, cupram-

\footnotetext{
$5 \mathrm{~J}$. G. Wiegerink, The moisture relations of textile fibers at elevated temperatures, J. Research NBS 24,645 (1940) RP1304.

${ }_{6}^{6}$. T. Mease, Measurement of the apparent fluidity of dispersions of cellulose in cuprammonium solution, J. Research NBS 22, 3 (1939) RP1179.

7 A. S. Tweedie, A study of the viscosity method for the determination of damage in silk, Can. J. Research 16B, 134-150 (1938).
} 
monium rayon, purified cotton, and mercerized cotton, were dyed with a solution containing 0.5 percent of benzopurpurine $4 B$ and 10 percent of $\mathrm{NaCl}$, based on the weight of the yarn. The heat-treated clothing and carpet wools were dyed with a solution containing 0.3 percent of alizarine astrole $B, 0.1$ percent of fast light yellow $2 G$, 0.25 percent of neolan red $3 B, 20$ percent of Glauber's salt, and 5 percent of sodium bisulfate. The dyed yarns were wound on dark cardboard and their spectral reflectances relative to magnesium oxide were determined at a wavelength of 501 millimicrons. This wavelength was chosen after a study of the complete reflectance curves (400 to $740 \lambda$ ) for typical samples.

The cystine contents of the heat-treated samples of clothing wool were determined by the Sullivan method. ${ }^{8}$

\section{RESULTS AND CONCLUSIONS}

Tables 1 to 9 give the breaking strengths, elongations at break, and "quality indices" of the yarns, after being heated at different humidities for various periods of time and then exposed to the standard conditions of 65-percent relative humidity and $70^{\circ} \mathrm{F}$ for at least 24 hours. Each value is the average of 25 tests. The "quality index" is the percentage of the original breaking strength after drying, multiplied by the percentage of the original elongation after drying. It appears to be useful in analyzing the results.

TABLE 1.-Viscose rayon yarn

\begin{tabular}{|c|c|c|c|c|c|c|}
\hline Temperature $\left({ }^{\circ} \mathrm{F}\right)$ & $\begin{array}{c}\text { Relative } \\
\text { humidity } 1\end{array}$ & $\begin{array}{c}\text { Absolute } \\
\text { humidity } 1\end{array}$ & $\begin{array}{l}\text { Time of } \\
\text { exposure }\end{array}$ & $\begin{array}{l}\text { Breaking } \\
\text { strength } \\
\text { (as \% of } \\
\text { original) }\end{array}$ & $\begin{array}{l}\text { Elonga- } \\
\text { tion } \\
\text { at break } \\
\text { (as \% of } \\
\text { original) }\end{array}$ & $\begin{array}{c}\text { Quality } \\
\text { index }\end{array}$ \\
\hline $221 \ldots$ & $\begin{array}{c}\% \\
1\end{array}$ & $\begin{array}{c}\% \\
1\end{array}$ & $\left\{\begin{array}{cc}h r & \\
& 1 \\
& 11 / 2 \\
& 6\end{array} \mid\right.$ & $\begin{array}{l}95 \\
94 \\
90\end{array}$ & $\begin{array}{l}97 \\
97 \\
89\end{array}$ & $\begin{array}{l}92 \\
91 \\
80\end{array}$ \\
\hline $221 \ldots$ & 48 & 57 & $\begin{array}{l}11 / 2 \\
3 \\
6\end{array}$ & $\begin{array}{r}99 \\
100 \\
97\end{array}$ & $\begin{array}{l}93 \\
91 \\
84\end{array}$ & $\begin{array}{l}92 \\
91 \\
81\end{array}$ \\
\hline $221 \ldots$ & 79 & 94 & $\begin{array}{l}11 / 2 \\
4 \\
6\end{array}$ & $\begin{array}{l}96 \\
97 \\
90\end{array}$ & $\begin{array}{l}97 \\
91 \\
83\end{array}$ & $\begin{array}{l}93 \\
88 \\
75\end{array}$ \\
\hline $257 \ldots$ & 0.5 & 1 & $\begin{array}{c}1 / 2 \\
11 / 2 \\
6\end{array}$ & $\begin{array}{l}92 \\
85 \\
72\end{array}$ & $\begin{array}{l}91 \\
79 \\
67\end{array}$ & $\begin{array}{l}84 \\
67 \\
48\end{array}$ \\
\hline $257 \ldots$ & 24 & 55 & $\begin{array}{l}3 / 4 \\
134 \\
6\end{array}$ & $\begin{array}{l}96 \\
85 \\
72\end{array}$ & $\begin{array}{l}86 \\
72 \\
56\end{array}$ & $\begin{array}{l}82 \\
61 \\
40\end{array}$ \\
\hline $257 \ldots$ & 42 & 96 & $\begin{array}{c}1 / 1 \\
6^{1 / 2}\end{array}$ & $\begin{array}{l}99 \\
90 \\
68\end{array}$ & $\begin{array}{l}93 \\
82 \\
57\end{array}$ & $\begin{array}{l}92 \\
74 \\
38\end{array}$ \\
\hline 302 & 0.2 & 0.5 & $\begin{array}{l}1 / 2 \\
1^{11 / 2}\end{array}$ & $\begin{array}{l}73 \\
67 \\
50\end{array}$ & $\begin{array}{l}61 \\
58 \\
42\end{array}$ & $\begin{array}{l}45 \\
39 \\
21\end{array}$ \\
\hline $302 \ldots$ & 12 & 56 & $\begin{array}{c}1 / 2 \\
6^{1 / 2}\end{array}$ & $\begin{array}{l}69 \\
57 \\
45\end{array}$ & $\begin{array}{l}54 \\
38 \\
23\end{array}$ & $\begin{array}{l}37 \\
22 \\
10\end{array}$ \\
\hline $302 \ldots$ & 20 & 96 & $6^{1 / 2}$ & $\begin{array}{l}83 \\
60 \\
43\end{array}$ & $\begin{array}{l}70 \\
50 \\
27\end{array}$ & $\begin{array}{l}58 \\
30 \\
11\end{array}$ \\
\hline
\end{tabular}

1 See footnote 4 of text.

${ }^{8}$ M. X. Sullivan, Public Health Reports 86 (1930). 
TABLE 2.-Cuprammonium rayon yarn

\begin{tabular}{|c|c|c|c|c|c|c|}
\hline Temperature $\left({ }^{\circ} \mathrm{F}\right)$ & $\begin{array}{l}\text { Relative } \\
\text { humidity }\end{array}$ & $\begin{array}{l}\text { Absolute } \\
\text { humidity }\end{array}$ & $\begin{array}{c}\text { Time of } \\
\text { exposure }\end{array}$ & $\begin{array}{l}\text { Breaking } \\
\text { strength } \\
\text { (as \% of } \\
\text { original) }\end{array}$ & \begin{tabular}{|c|} 
Elongation \\
at break \\
(as \% of \\
original)
\end{tabular} & $\begin{array}{c}\text { Quality } \\
\text { index }\end{array}$ \\
\hline $221 \ldots$ & $\begin{array}{l}\% \\
1\end{array}$ & $\begin{array}{l}\% \\
1\end{array}$ & $\begin{array}{l}h r \\
1 \\
2 \\
6\end{array}$ & $\begin{array}{l}100 \\
100 \\
100\end{array}$ & $\begin{array}{l}94 \\
94 \\
94\end{array}$ & $\begin{array}{l}94 \\
94 \\
94\end{array}$ \\
\hline 221 & 48 & 57 & $\begin{array}{l}11 / 2 \\
3 \\
6\end{array}$ & $\begin{array}{l}97 \\
94 \\
86\end{array}$ & $\begin{array}{l}85 \\
76 \\
67\end{array}$ & $\begin{array}{l}82 \\
71 \\
58\end{array}$ \\
\hline $221 \ldots$ & 79 & 94 & $\begin{array}{l}2 \\
4 \\
6\end{array}$ & $\begin{array}{l}98 \\
96 \\
92\end{array}$ & $\begin{array}{l}79 \\
61 \\
58\end{array}$ & $\begin{array}{l}77 \\
59 \\
53\end{array}$ \\
\hline $257 \ldots$ & 0.5 & 1 & $\begin{array}{l}1^{1 / 2} \\
1^{11 / 2}\end{array}$ & $\begin{array}{c}100 \\
96 \\
96\end{array}$ & $\begin{array}{l}97 \\
91 \\
85\end{array}$ & $\begin{array}{l}97 \\
87 \\
82\end{array}$ \\
\hline 257 & 24 & 55 & $\begin{array}{c}34 \\
134 \\
6\end{array}$ & $\begin{array}{l}97 \\
89 \\
77\end{array}$ & $\begin{array}{l}85 \\
70 \\
55\end{array}$ & $\begin{array}{l}82 \\
62 \\
42\end{array}$ \\
\hline 257 & 42 & 96 & $\begin{array}{l}1 \\
2 \\
6\end{array}$ & $\begin{array}{l}98 \\
87 \\
76\end{array}$ & $\begin{array}{l}79 \\
58 \\
45\end{array}$ & $\begin{array}{l}77 \\
50 \\
34\end{array}$ \\
\hline 302 & 0.2 & 0.5 & $\begin{array}{l}{ }^{1 / 2} \\
1^{1 / 2}\end{array}$ & $\begin{array}{l}94 \\
93 \\
76\end{array}$ & $\begin{array}{l}79 \\
73 \\
55\end{array}$ & $\begin{array}{l}74 \\
68 \\
42\end{array}$ \\
\hline 302 & 12 & 56 & $\begin{array}{l}1 / 2 \\
1^{11 / 2} \\
6^{1 / 2}\end{array}$ & $\begin{array}{l}89 \\
79 \\
65\end{array}$ & $\begin{array}{l}73 \\
60 \\
36\end{array}$ & $\begin{array}{l}65 \\
47 \\
23\end{array}$ \\
\hline 302 & 20 & 96 & $\begin{array}{l}1 / 2 \\
1^{13 / 2} \\
6\end{array}$ & $\begin{array}{l}86 \\
71 \\
55 \\
\end{array}$ & $\begin{array}{l}67 \\
42 \\
24 \\
\end{array}$ & $\begin{array}{l}58 \\
30 \\
13\end{array}$ \\
\hline
\end{tabular}

TABLE 3.-Clothing wool yarn

\begin{tabular}{|c|c|c|c|c|c|c|c|}
\hline 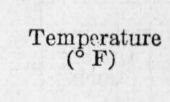 & $\begin{array}{c}\text { Relative } \\
\text { humidity }\end{array}$ & $\begin{array}{l}\text { Absolute } \\
\text { humidity }\end{array}$ & $\begin{array}{l}\text { Time of } \\
\text { exposure }\end{array}$ & $\begin{array}{l}\text { Breaking } \\
\text { strength } \\
\text { (as \% of } \\
\text { original) }\end{array}$ & $\begin{array}{c}\text { Elongation } \\
\text { at break } \\
\text { (as \% of } \\
\text { original) }\end{array}$ & $\begin{array}{c}\text { Quality } \\
\text { index }\end{array}$ & $\begin{array}{l}\text { Cystine } \\
\text { content } \\
\text { (original } \\
=11.5 \text { ) }\end{array}$ \\
\hline $221 \ldots$ & $\begin{array}{l}\% \\
1\end{array}$ & $\begin{array}{r}\% \\
1\end{array}$ & $h r \begin{array}{r}1 \\
2 \\
2 \\
6\end{array}$ & $\begin{array}{r}102 \\
99 \\
95\end{array}$ & $\begin{array}{r}100 \\
100 \\
93\end{array}$ & $\begin{array}{r}102 \\
99 \\
88\end{array}$ & $\begin{array}{l}\% \\
11.3\end{array}$ \\
\hline $221 \ldots$ & 48 & 57 & $\begin{array}{l}11 / 2 \\
3 \\
6\end{array}$ & $\begin{array}{l}95 \\
91 \\
91\end{array}$ & $\begin{array}{l}97 \\
91 \\
91\end{array}$ & $\begin{array}{l}92 \\
83 \\
83\end{array}$ & 11.2 \\
\hline $221 \ldots$ & 79 & 94 & $\begin{array}{l}2 \\
4 \\
6\end{array}$ & $\begin{array}{r}100 \\
97 \\
95\end{array}$ & $\begin{array}{r}100 \\
89 \\
86\end{array}$ & $\begin{array}{r}100 \\
86 \\
82\end{array}$ & 9.6 \\
\hline $257 \ldots$ & 0.5 & 1 & $\begin{array}{l}1 / 2 \\
11 / 2 \\
6\end{array}$ & $\begin{array}{r}100 \\
91 \\
91\end{array}$ & $\begin{array}{r}100 \\
93 \\
93\end{array}$ & $\begin{array}{r}100 \\
85 \\
85\end{array}$ & 11.3 \\
\hline $257 \ldots$ & 24 & 55 & $\begin{array}{l}3 / 4 \\
13 / 4 \\
6\end{array}$ & $\begin{array}{r}105 \\
97 \\
95\end{array}$ & $\begin{array}{l}98 \\
97 \\
93\end{array}$ & $\begin{array}{r}103 \\
94 \\
88\end{array}$ & 11. \\
\hline $257 \ldots$ & 42 & 96 & $\begin{array}{l}1 \\
2 \\
6\end{array}$ & $\begin{array}{r}100 \\
98 \\
94\end{array}$ & $\begin{array}{l}98 \\
93 \\
89\end{array}$ & $\begin{array}{l}98 \\
91 \\
84\end{array}$ & 9.5 \\
\hline 302 & & 0.5 & $6^{1 / 2}$ & $\begin{array}{l}99 \\
92 \\
91\end{array}$ & $\begin{array}{r}100 \\
97 \\
93\end{array}$ & $\begin{array}{l}99 \\
89 \\
85\end{array}$ & 10.3 \\
\hline $302 \ldots$ & 12 & 56 & $\begin{array}{l}1 / 2 \\
11 / 2 \\
6\end{array}$ & $\begin{array}{r}107 \\
93 \\
91\end{array}$ & $\begin{array}{l}93 \\
91 \\
91\end{array}$ & $\begin{array}{r}100 \\
85 \\
83\end{array}$ & 10.3 \\
\hline $302 \ldots$ & 20 & 96 & $\begin{array}{l}1 / 2 \\
11 / 2 \\
6\end{array}$ & $\begin{array}{r}100 \\
96 \\
90\end{array}$ & $\begin{array}{l}97 \\
93 \\
85\end{array}$ & $\begin{array}{l}97 \\
89 \\
77\end{array}$ & 8.8 \\
\hline
\end{tabular}


TABLE 4.-Carpet wool yarn

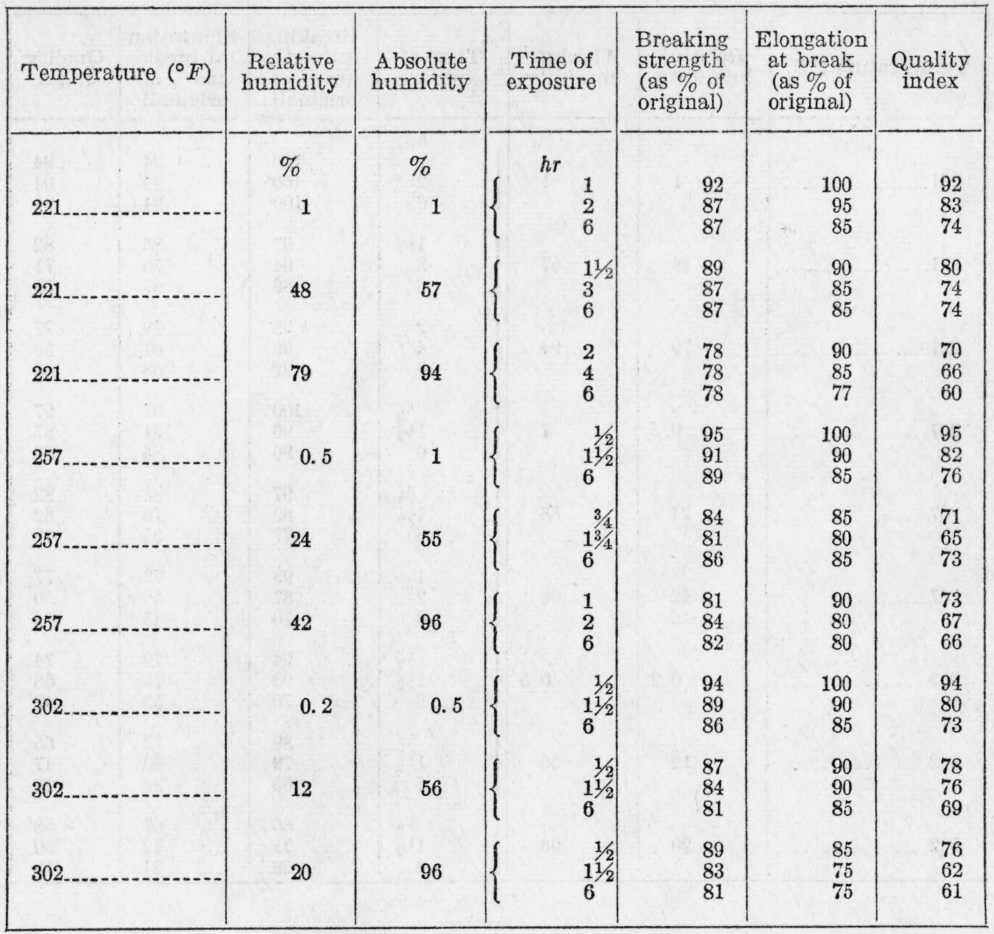

TABLE 5.- "Purified" cotton yarn

\begin{tabular}{|c|c|c|c|c|c|c|}
\hline Temperature $\left({ }^{\circ} F\right)$ & $\begin{array}{l}\text { Relative } \\
\text { humidity }\end{array}$ & $\begin{array}{l}\text { Absolute } \\
\text { humidity }\end{array}$ & $\begin{array}{l}\text { Time of } \\
\text { exposure }\end{array}$ & $\begin{array}{l}\text { Breaking } \\
\text { strength } \\
\text { (as \% of of } \\
\text { original) }\end{array}$ & $\begin{array}{l}\text { Elongation } \\
\text { at break } \\
\text { (as \% of } \\
\text { original) }\end{array}$ & $\begin{array}{l}\text { Quality } \\
\text { index }\end{array}$ \\
\hline $221 \ldots$ & $\%_{1}$ & ${ }_{1}{ }_{1}$ & $\begin{array}{l}h r \\
1 \\
6\end{array}$ & $\begin{array}{l}99 \\
95\end{array}$ & $\begin{array}{l}97 \\
97\end{array}$ & $\begin{array}{l}96 \\
92\end{array}$ \\
\hline $221 \ldots$ & 48 & 57 & $\begin{array}{l}1 \\
6\end{array}$ & $\begin{array}{r}100 \\
93\end{array}$ & $\begin{array}{l}98 \\
91\end{array}$ & $\begin{array}{l}98 \\
85\end{array}$ \\
\hline 221. & 79 & 94 & $\begin{array}{l}1 \\
6\end{array}$ & $\begin{array}{l}91 \\
87\end{array}$ & $\begin{array}{l}94 \\
89\end{array}$ & $\begin{array}{l}86 \\
77\end{array}$ \\
\hline $257 \ldots$ & 0.5 & 1 & $\begin{array}{l}1 \\
6\end{array}$ & $\begin{array}{r}100 \\
92\end{array}$ & $\begin{array}{r}102 \\
93\end{array}$ & $\begin{array}{r}102 \\
86\end{array}$ \\
\hline $257 \ldots$ & 24 & 55 & 6 & $\begin{array}{l}93 \\
85\end{array}$ & $\begin{array}{l}89 \\
87\end{array}$ & $\begin{array}{l}83 \\
74\end{array}$ \\
\hline 257 & 42 & 96 & $\begin{array}{l}1 \\
6\end{array}$ & $\begin{array}{l}87 \\
78\end{array}$ & $\begin{array}{l}90 \\
81\end{array}$ & $\begin{array}{l}78 \\
63\end{array}$ \\
\hline $302 \ldots$ & 0.2 & 0.5 & $\begin{array}{l}1 \\
1\end{array}$ & $\begin{array}{l}98 \\
94 \\
84\end{array}$ & $\begin{array}{r}95 \\
\quad 93 \\
82\end{array}$ & $\begin{array}{l}93 \\
87 \\
69\end{array}$ \\
\hline $302 \ldots$ & 12 & 56 & $\begin{array}{l}1 \\
6\end{array}$ & $\begin{array}{l}92 \\
79 \\
68\end{array}$ & $\begin{array}{l}90 \\
80 \\
67\end{array}$ & $\begin{array}{l}8 \mathbf{3} \\
63 \\
46\end{array}$ \\
\hline $302 \ldots$ & 20 & 96 & $\begin{array}{l}3 \\
13\end{array}$ & $\begin{array}{l}96 \\
82 \\
58\end{array}$ & $\begin{array}{r}100 \\
85 \\
69\end{array}$ & $\begin{array}{l}96 \\
70 \\
40\end{array}$ \\
\hline
\end{tabular}


TABLE 6.-Raw cotton yarn

\begin{tabular}{|c|c|c|c|c|c|c|}
\hline Temperature $\left({ }^{\circ} F\right)$ & $\begin{array}{l}\text { Relative } \\
\text { humidity }\end{array}$ & $\begin{array}{l}\text { Absolute } \\
\text { humidity }\end{array}$ & $\begin{array}{l}\text { Time of } \\
\text { exposure }\end{array}$ & $\begin{array}{l}\text { Breaking } \\
\text { strength } \\
\text { (as \% of } \\
\text { original) }\end{array}$ & $\begin{array}{l}\text { Elongation } \\
\text { at break } \\
\text { (as \% of } \\
\text { original) }\end{array}$ & $\begin{array}{l}\text { Quality } \\
\text { index }\end{array}$ \\
\hline 221 & $\begin{array}{l}\%_{1} \\
\end{array}$ & $\begin{array}{c}\% \\
1\end{array}$ & $\begin{array}{ll}h r & 11 \\
& 6\end{array}$ & $\begin{array}{l}95 \\
94\end{array}$ & $\begin{array}{l}94 \\
93\end{array}$ & $\begin{array}{l}89 \\
87\end{array}$ \\
\hline 221 & 48 & 57 & $\begin{array}{l}11 \\
6\end{array}$ & $\begin{array}{l}89 \\
92\end{array}$ & $\begin{array}{r}100 \\
95\end{array}$ & $\begin{array}{l}89 \\
87\end{array}$ \\
\hline 221 & 79 & 94 & $\begin{array}{l}11 \\
6\end{array}$ & $\begin{array}{l}93 \\
95\end{array}$ & $\begin{array}{l}95 \\
92\end{array}$ & $\begin{array}{l}88 \\
87\end{array}$ \\
\hline 257 & 0.5 & 1 & $\begin{array}{l}11 \\
6\end{array}$ & $\begin{array}{l}89 \\
88\end{array}$ & $\begin{array}{l}90 \\
90\end{array}$ & $\begin{array}{l}80 \\
79\end{array}$ \\
\hline 257 & 24 & 55 & $\begin{array}{l}11 \\
6\end{array}$ & $\begin{array}{l}98 \\
93\end{array}$ & $\begin{array}{l}95 \\
90\end{array}$ & $\begin{array}{l}93 \\
84\end{array}$ \\
\hline 257 & 42 & 96 & $\begin{array}{l}11 \\
6\end{array}$ & $\begin{array}{l}90 \\
92\end{array}$ & $\begin{array}{l}90 \\
88\end{array}$ & $\begin{array}{l}81 \\
81\end{array}$ \\
\hline 302 & 0.2 & 0.5 & $y_{1}^{1}$ & $\begin{array}{l}96 \\
94 \\
89\end{array}$ & $\begin{array}{l}90 \\
90 \\
84\end{array}$ & $\begin{array}{l}86 \\
85 \\
75\end{array}$ \\
\hline 302 & 12 & 56 & $\begin{array}{c}3 \\
1^{13} \\
6\end{array}$ & $\begin{array}{l}94 \\
91 \\
89\end{array}$ & $\begin{array}{l}90 \\
85 \\
80\end{array}$ & $\begin{array}{l}85 \\
77 \\
71\end{array}$ \\
\hline $302 \ldots$ & 20 & 96 & $\begin{array}{l}\frac{1}{1} \\
1^{11}\end{array}$ & $\begin{array}{l}85 \\
89 \\
84\end{array}$ & $\begin{array}{l}90 \\
86 \\
83\end{array}$ & $\begin{array}{l}77 \\
77 \\
70\end{array}$ \\
\hline
\end{tabular}

TABLE 7.-Mercerized cotton yarn

\begin{tabular}{|c|c|c|c|c|c|c|}
\hline Temperature $\left({ }^{\circ} F\right)$ & $\begin{array}{l}\text { Relative } \\
\text { humidity }\end{array}$ & $\begin{array}{l}\text { Absolute } \\
\text { humidity }\end{array}$ & $\begin{array}{l}\text { Time of } \\
\text { exposure }\end{array}$ & $\begin{array}{l}\text { Breaking } \\
\text { strength } \\
\text { (as \% of } \\
\text { original) }\end{array}$ & $\begin{array}{l}\text { Elongation } \\
\text { at break } \\
\text { as \% of } \\
\text { original) }\end{array}$ & $\begin{array}{l}\text { Quality } \\
\text { index }\end{array}$ \\
\hline 221 & $\begin{array}{r}\% \\
1\end{array}$ & $\begin{array}{r}\% \\
1\end{array}$ & $\begin{array}{ll}h r & \\
1 \\
2 \\
& 6\end{array}$ & $\begin{array}{r}91 \\
92 \\
108\end{array}$ & $\begin{array}{r}90 \\
95 \\
105\end{array}$ & $\begin{array}{r}82 \\
87 \\
113\end{array}$ \\
\hline $221 \ldots$ & 48 & 57 & $\begin{array}{l}l^{11 / 2} \\
6\end{array}$ & $\begin{array}{r}92 \\
98 \\
105\end{array}$ & $\begin{array}{l}95 \\
96 \\
97\end{array}$ & $\begin{array}{r}87 \\
94 \\
102\end{array}$ \\
\hline $221 \ldots$ & 79 & 94 & $\begin{array}{l}2 \\
4 \\
6\end{array}$ & $\begin{array}{l}92 \\
97 \\
94\end{array}$ & $\begin{array}{l}94 \\
95 \\
93\end{array}$ & $\begin{array}{l}86 \\
92 \\
87\end{array}$ \\
\hline $257 \ldots$ & 0.5 & 1 & $\begin{array}{c}1 / 2 \\
1^{1 / 2}\end{array}$ & $\begin{array}{l}96 \\
92 \\
99\end{array}$ & $\begin{array}{l}96 \\
94 \\
96\end{array}$ & $\begin{array}{l}92 \\
86 \\
95\end{array}$ \\
\hline $257 \ldots$ & 24 & 55 & $\begin{array}{c}3 / 4 \\
13 \\
6\end{array}$ & $\begin{array}{r}97 \\
88 \\
100\end{array}$ & $\begin{array}{l}96 \\
90 \\
98\end{array}$ & $\begin{array}{l}93 \\
79 \\
98\end{array}$ \\
\hline $257 \ldots$ & 42 & 96 & $\begin{array}{l}1 \\
2 \\
6\end{array}$ & $\begin{array}{l}87 \\
92 \\
88\end{array}$ & $\begin{array}{l}92 \\
90 \\
89\end{array}$ & $\begin{array}{l}80 \\
83 \\
78\end{array}$ \\
\hline $302 \ldots$ & 0.2 & 0.5 & $\begin{array}{l}1 / 2 \\
13 / 2 \\
6\end{array}$ & $\begin{array}{l}94 \\
90 \\
92\end{array}$ & $\begin{array}{l}93 \\
91 \\
95\end{array}$ & $\begin{array}{l}87 \\
82 \\
87\end{array}$ \\
\hline $302 \ldots$ & 12 & 56 & $\begin{array}{c}1 / 2 \\
11 / 2 \\
6\end{array}$ & $\begin{array}{l}97 \\
87 \\
91\end{array}$ & $\begin{array}{l}95 \\
89 \\
93\end{array}$ & $\begin{array}{l}92 \\
77 \\
85\end{array}$ \\
\hline $302 \ldots$ & 20 & 96 & $\begin{array}{l}31 / 2 \\
11 / 2 \\
6\end{array}$ & $\begin{array}{l}93 \\
88 \\
89\end{array}$ & $\begin{array}{l}90 \\
89 \\
89\end{array}$ & $\begin{array}{l}84 \\
78 \\
79\end{array}$ \\
\hline
\end{tabular}

$259104-40-4$ 
TABLE 8.-Degummed silk yarn

\begin{tabular}{|c|c|c|c|c|c|c|}
\hline Temperature $\left({ }^{\circ} \mathrm{F}\right)$ & $\begin{array}{l}\text { Relative } \\
\text { humidity }\end{array}$ & $\begin{array}{l}\text { Absolute } \\
\text { humidity }\end{array}$ & $\begin{array}{l}\text { Time of } \\
\text { exposure }\end{array}$ & $\begin{array}{l}\text { Breaking } \\
\text { strength } \\
\text { (as \% of of } \\
\text { original) }\end{array}$ & $\begin{array}{l}\text { Elongation } \\
\text { at break } \\
\text { (as o of } \\
\text { original) }\end{array}$ & $\begin{array}{l}\text { Quality } \\
\text { index }\end{array}$ \\
\hline $221 \ldots$ & $\begin{array}{l}\% \\
1\end{array}$ & $\begin{array}{l}\% \\
1\end{array}$ & $\begin{array}{rr}h r & \\
& 1 \\
& 2 \\
& 6\end{array}$ & $\begin{array}{l}96 \\
96 \\
96\end{array}$ & $\begin{array}{l}100 \\
100 \\
100\end{array}$ & $\begin{array}{l}96 \\
96 \\
96\end{array}$ \\
\hline 221. & 48 & 57 & $\begin{array}{l}11 / 2 \\
3 \\
6\end{array}$ & $\begin{array}{r}100 \\
98 \\
93\end{array}$ & $\begin{array}{l}92 \\
88 \\
90\end{array}$ & $\begin{array}{l}92 \\
86 \\
84\end{array}$ \\
\hline 221 . & 79 & 94 & $\begin{array}{l}2 \\
4 \\
6\end{array}$ & $\begin{array}{l}99 \\
99 \\
99\end{array}$ & $\begin{array}{l}92 \\
92 \\
90\end{array}$ & $\begin{array}{l}91 \\
91 \\
89\end{array}$ \\
\hline $257 \ldots$ & 0.5 & 1 & $\begin{array}{l}1^{1 / 2} \\
1^{1 / 2}\end{array}$ & $\begin{array}{l}99 \\
96 \\
93\end{array}$ & $\begin{array}{l}99 \\
93 \\
88\end{array}$ & $\begin{array}{l}98 \\
89 \\
82\end{array}$ \\
\hline 257. & 24 & 55 & $\begin{array}{l}\frac{3 / 4}{4} \\
1^{3 / 2}\end{array}$ & $\begin{array}{l}94 \\
91 \\
89\end{array}$ & $\begin{array}{l}85 \\
84 \\
81\end{array}$ & $\begin{array}{l}80 \\
76 \\
72\end{array}$ \\
\hline 257. & 42 & 96 & $\begin{array}{l}1 \\
2 \\
6\end{array}$ & $\begin{array}{r}100 \\
99 \\
89\end{array}$ & $\begin{array}{l}86 \\
84 \\
78\end{array}$ & $\begin{array}{l}86 \\
83 \\
69\end{array}$ \\
\hline $302 \ldots$ & 0.2 & 0.5 & $\begin{array}{l}1 / 2 \\
1^{13 / 2}\end{array}$ & $\begin{array}{l}90 \\
88 \\
74\end{array}$ & $\begin{array}{l}89 \\
84 \\
75\end{array}$ & $\begin{array}{l}80 \\
74 \\
56\end{array}$ \\
\hline $302 \ldots$ & 12 & 56 & $\begin{array}{l}1 / 2 \\
11 / 2 \\
6\end{array}$ & $\begin{array}{l}93 \\
92 \\
76\end{array}$ & $\begin{array}{l}98 \\
85 \\
74\end{array}$ & $\begin{array}{l}91 \\
78 \\
56\end{array}$ \\
\hline $302 \ldots$ & 20 & 96 & $\begin{array}{l}1 / 2 \\
1^{1 / 2}\end{array}$ & $\begin{array}{l}96 \\
95 \\
76\end{array}$ & $\begin{array}{l}91 \\
89 \\
74\end{array}$ & $\begin{array}{l}87 \\
85 \\
56\end{array}$ \\
\hline
\end{tabular}

TABLE 9.-Acetate yarn

\begin{tabular}{|c|c|c|c|c|c|c|}
\hline Temperature $\left({ }^{\circ} \mathrm{F}\right)$ & $\begin{array}{c}\text { Relative } \\
\text { humidity }\end{array}$ & $\begin{array}{l}\text { Absolute } \\
\text { humidity }\end{array}$ & $\begin{array}{l}\text { Time of } \\
\text { exposure }\end{array}$ & $\begin{array}{l}\text { Breaking } \\
\text { strength } \\
\text { (as \% of } \\
\text { original) }\end{array}$ & $\begin{array}{l}\text { Elongation } \\
\text { at break } \\
\text { (as \% of } \\
\text { original) }\end{array}$ & $\begin{array}{c}\text { Quality } \\
\text { index }\end{array}$ \\
\hline 221 & $\begin{array}{r}\% \\
1\end{array}$ & $\begin{array}{l}\% \\
1\end{array}$ & $h r \begin{array}{l}1 \\
\\
\\
\\
\\
\\
6\end{array}$ & $\begin{array}{r}100 \\
94 \\
94\end{array}$ & $\begin{array}{l}98 \\
95 \\
95\end{array}$ & $\begin{array}{l}98 \\
89 \\
89\end{array}$ \\
\hline 221 & 48 & 57 & $\begin{array}{l}11 / 2 \\
3 \\
6\end{array}$ & $\begin{array}{l}98 \\
98 \\
98\end{array}$ & $\begin{array}{l}90 \\
93 \\
93\end{array}$ & $\begin{array}{l}88 \\
91 \\
91\end{array}$ \\
\hline 221 & 79 & 94 & $\begin{array}{l}2 \\
4 \\
6\end{array}$ & $\begin{array}{l}82 \\
83 \\
82\end{array}$ & $\begin{array}{l}84 \\
85 \\
84\end{array}$ & $\begin{array}{l}69 \\
70 \\
69\end{array}$ \\
\hline $257 \ldots$ & 0.5 & 1 & $\begin{array}{l}1 / 2 / 2 \\
1^{11} / 2\end{array}$ & $\begin{array}{l}99 \\
96 \\
95\end{array}$ & $\begin{array}{l}95 \\
91 \\
95\end{array}$ & $\begin{array}{l}94 \\
87 \\
90\end{array}$ \\
\hline 257. & 24 & 55 & $\begin{array}{l}3 / 4 / 4 \\
1^{3 / 3} \\
6\end{array}$ & $\begin{array}{l}99 \\
98 \\
93\end{array}$ & $\begin{array}{l}95 \\
95 \\
92\end{array}$ & $\begin{array}{l}94 \\
93 \\
86\end{array}$ \\
\hline 257 & 42 & 96 & $\begin{array}{l}1 \\
2 \\
6\end{array}$ & $\begin{array}{r}100 \\
99 \\
100\end{array}$ & $\begin{array}{l}87 \\
84 \\
86\end{array}$ & $\begin{array}{l}87 \\
83 \\
86\end{array}$ \\
\hline 302 & 0.2 & 0.5 & $\begin{array}{l}1 / 2 \\
1^{1} / 2\end{array}$ & $\begin{array}{r}98 \\
100 \\
100\end{array}$ & $\begin{array}{l}95 \\
95 \\
92\end{array}$ & $\begin{array}{l}93 \\
95 \\
92\end{array}$ \\
\hline 302 & 12 & 56 & $\begin{array}{l}1 / 2 \\
1^{1} / 2\end{array}$ & $\begin{array}{r}100 \\
98 \\
96\end{array}$ & $\begin{array}{l}91 \\
91 \\
88\end{array}$ & $\begin{array}{l}91 \\
89 \\
84\end{array}$ \\
\hline 302 & 20 & 96 & $\begin{array}{l}1^{1 / 2} / 2 \\
1^{1 / 2}\end{array}$ & $\begin{array}{r}103 \\
102 \\
96\end{array}$ & $\begin{array}{l}87 \\
85 \\
84\end{array}$ & $\begin{array}{l}90 \\
87 \\
81\end{array}$ \\
\hline
\end{tabular}


The relationships between temperature and humidity of drying, on the one hand, and quality index, fluidity, moisture content, and affinity for dyes of the dried conditioned fibers, on the other hand, are shown in figures 1 to 4 , respectively. The data are for the 6 -hour treatment, the maximum time of exposure used in the experiments. The solid lines in the figures indicate the averages of the values for the different humidities at constant temperature. These lines show in a general way the effects of heat, while the departures of the points show the effects of humidity. It should be emphasized that the results and conclusions based on them apply only to the samples examined. Further work is necessary to determine what variation is to be expected in different lots of the same fiber, or when the fibers contain oils, sizing materials, and dyes.

Figure 1 shows that the quality indices of all of the yarns were adversely affected by the temperatures at which the yarns were heated. All were decreased by heating for 6 hours at $221^{\circ} \mathrm{F}$, and the lowering of the quality indices for the viscose and cuprammonium rayons, the degummed silk, and the cotton yarns was greater as the temperature was increased. Heating in air of an absolute humidity of approximately 95 percent tended to give lower quality indices for all of the yarns, except the raw cotton, than heating in air of an absolute humidity of approximately 1 percent. The greatest effect of humidity was obtained with the cuprammonium rayon, whose behavior was in marked contrast to that of the viscose rayon in this respect. Indeed, the effect of the humidity of the air on the quality index of cuprammonium rayon was as great as that of the change in temperature from $221^{\circ}$ to $302^{\circ} \mathrm{F}$.

The effects of heat on the clothing and carpet wool yarns were less than the effects of humidity as measured by the quality index, the average line being nearly horizontal. This is particularly true of the carpet wool yarn, which shows the greatest degradation at the high humidity. The results also indicate that the carpet wool is less resistant to heat and humidity than the corresponding clothing wool yarn.

Figure 1 indicates that heat had a greater effect on the quality indices of the purified and the raw cotton yarns than on the wool yarns. The purified cotton yarn was less resistant to heat treatment than the raw cotton yarn. The high humidity had a more degrading effect at constant temperature on the purified cotton yarn than the low humidity, but the raw cotton yarn was scarcely affected. This difference in behavior between the raw and the purified cotton yarms may be attributed to a protective action of the natural waxes in the raw cotton, which are removed by the caustic purification.

It appears from the results for the mercerized cotton and the acetate yarns that the effect of humidity, particularly the high humidity, which had the greatest degrading effect, was relatively more important than the effect of heat. In fact, the high humidity at $221^{\circ}$ $\mathrm{F}$ gave the lowest quality index for the acetate yarn.

This low quality index for the acetate yarn dried at $221^{\circ} \mathrm{F}$ at the high humidity may be explained by the data given in table 9 . The breaking strength of the acetate yarn was found to decrease only when the yarn was heated at $221^{\circ} \mathrm{F}$ at the high humidity. At any of the other conditions studied there was no significant decrease in the breaking strength. On the other hand, there were appreciable 
variations in the elongation, which was lower at a high humidity at each temperature than at a low humidity. The yarns dried at the high humidity were more brittle than the yarns dried at the low
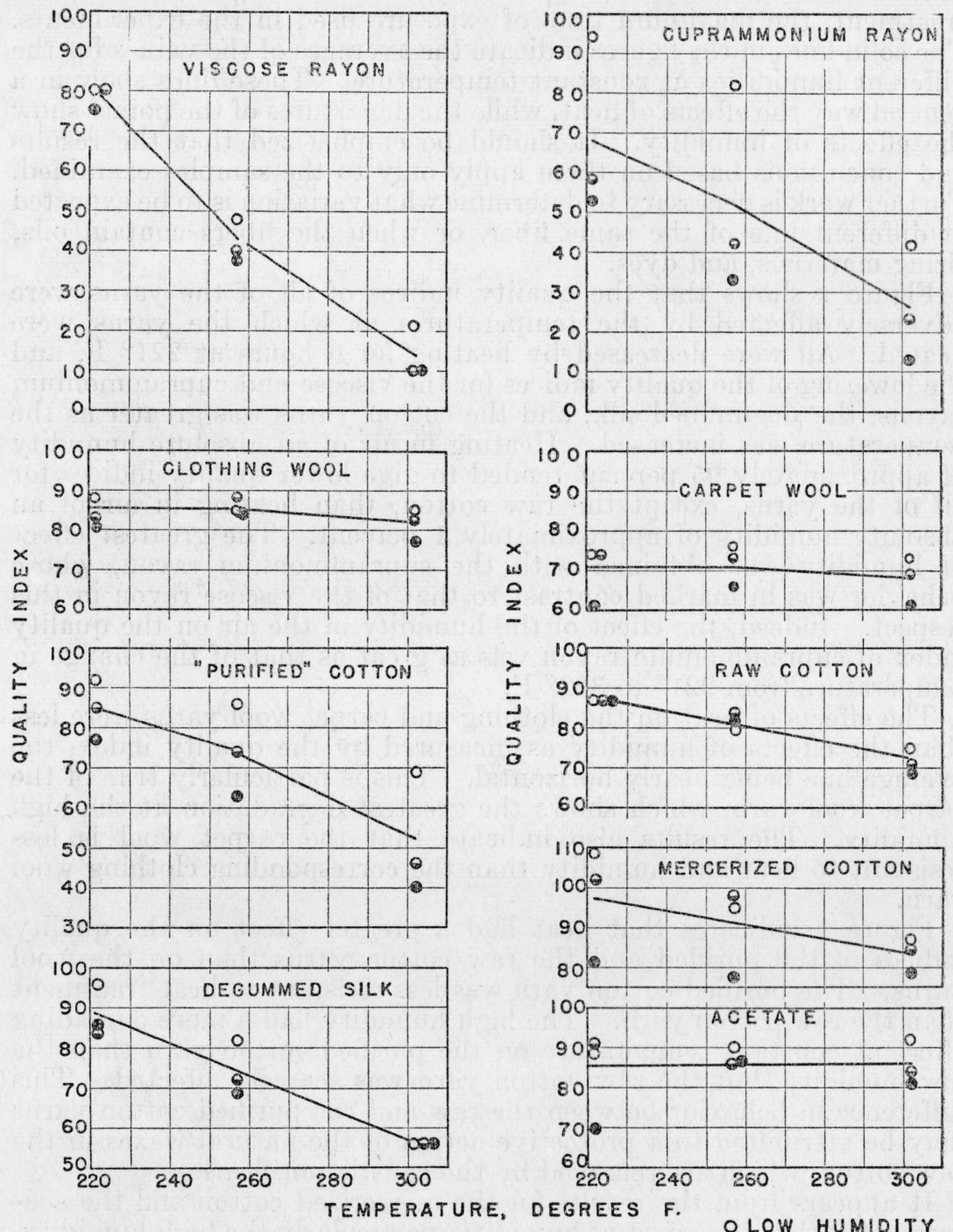

DEGREES F.

O LOW HUMIDITY

- MEDIUM HUMIDITY

- HIGH HUMIDITY

FigURE 1.-Relationship between quality index and temperature of drying at different humidities for 6-hour time of exposure.

humidity, even after conditioning them. The fact that there was a decrease in breaking strength of the yarns heated at $221^{\circ} \mathrm{F}$ at the high humidity may indicate that this particular condition aids hydrolysis. The water in the acetate yarn before drying would be evaporated more slowly at $221^{\circ} \mathrm{F}$ at the high humidity than at any 

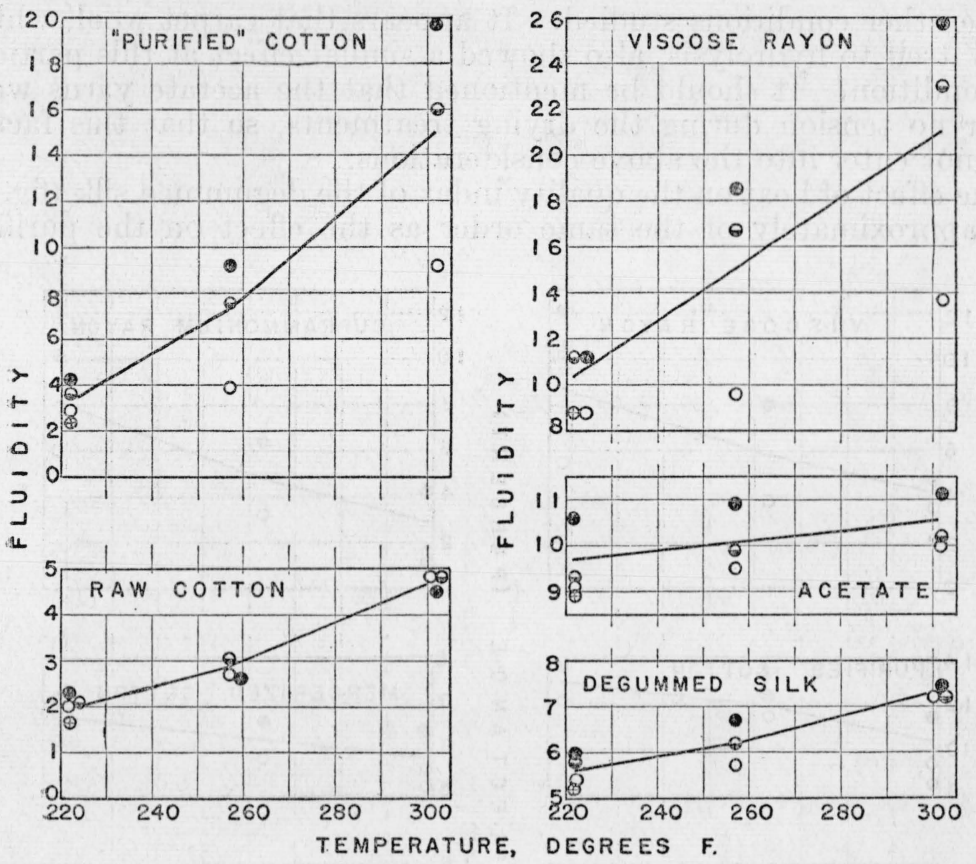

O LOW HUMIDITY

๑ MEDIUM HUMIDITY

- HIGH HUMIDITY

๑ ORIGINAL

FIGURE 2.-Relationship between fluidity and temperature of drying at different humidities for 6-hour time of exposure.


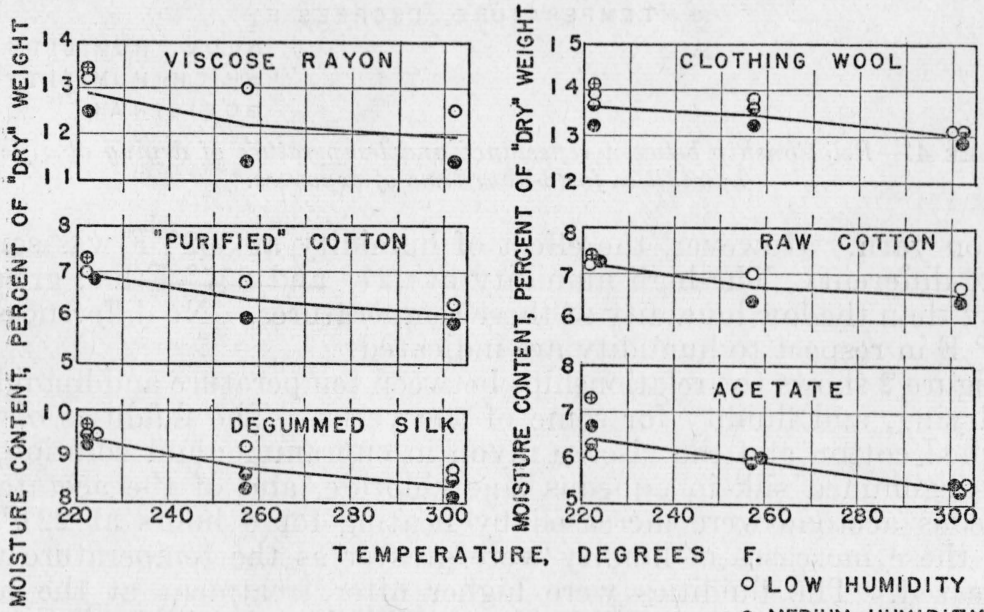

F.

O LOW HUMIDITY

Q MEDIUM HUMIDITY

- HIGH HUMIDITY

๑ ORIGINAL

FIGURE 3.-Relationship between moisture content on conditioning and temperature of drying at different humidities for 6-hour time of exposure. 
of the other conditions studied. It appears that carpet wool, which lends itself to hydrolysis, also showed a similar effect at this particular condition. It should be mentioned that the acetate yarns were under no tension during the drying treatments, so that this factor does not enter into the above considerations.

The effect of heat on the quality index of the degummed silk (fig. 1) was approximately of the same order as the effect on the purified
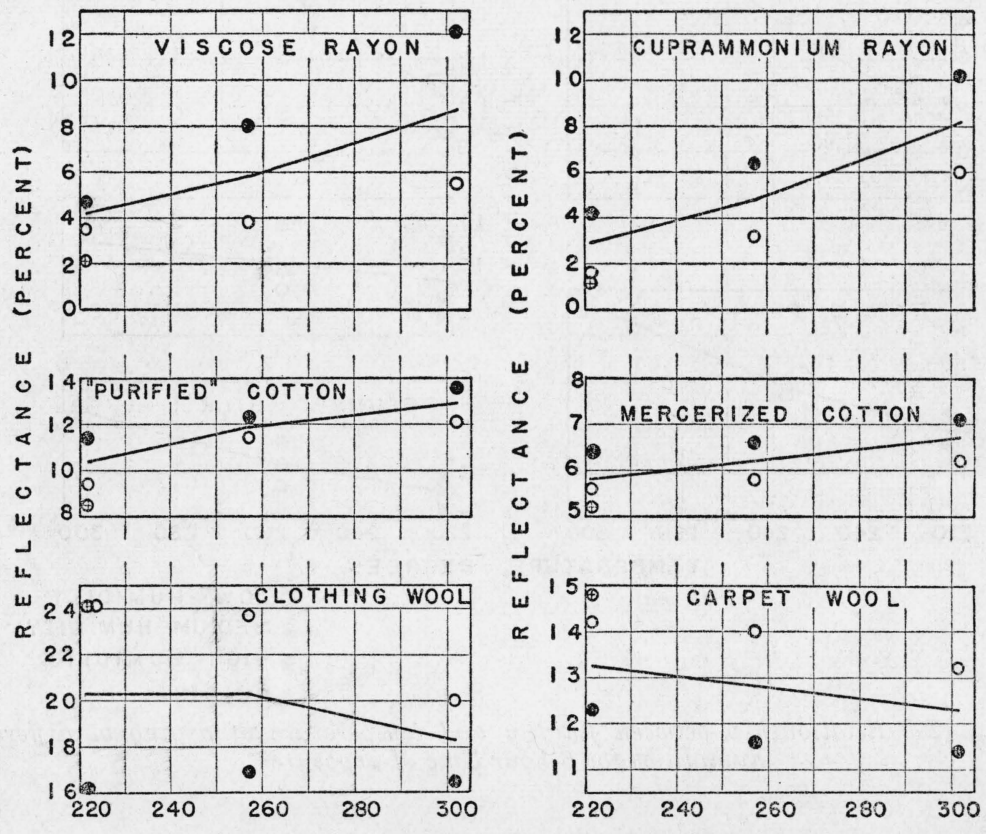

TEMPERATURE, DEGREES F.

OLOW HUMIDITY

๑HIGH HUMIDITY

OORIGINAL

FIGURE 4.-Relationship between reflectance and temperature of drying at different humidities for 6-hour time of exposure.

cotton yarn. However, the effect of humidity at $302^{\circ} \mathrm{F}$ was somewhat different. The high humidity at $221^{\circ}$ and $257^{\circ} \mathrm{F}$ had greater effect than the low humidity at these temperatures. No differences at $302^{\circ} \mathrm{F}$ in respect to humidity are indicated.

Figure 2 shows the relationships between temperature and humidity of drying, and fluidity for some of the yarns. The fluidities of the purified cotton and the viscose rayon in cuprammonium solution, of the degummed silk in aqueous zinc chloride, and of the acetate in aqueous acetone were increased by heating for 6 hours at $221^{\circ} \mathrm{F}$, and these increases in fluidity were greater as the temperature was increased. The fluidities were higher after treatment at the high humidity than at the low humidity. This increase in fluidity indicates greater degradation and corresponds well with the quality indices of the dried fibers mentioned above. The fluidities of the dried raw cotton in cuprammonium solution, however, did not change with 
change in humidity at constant temperature, an effect which was also found to be true for the quality indices.

Although the viscose rayon gave lower values of quality index for the high humidity at a given temperature, the differences were small. However, figure 2 indicates that the effect of humidity is important. The fluidities are much higher at the high humidity than at the low humidity, which indicates that at the temperatures studied the high humidity has a greater degrading effect than a low humidity.

Figure 3 shows the effects of heat and humidity on the moisture contents of the various textile materials when conditioned in an atmosphere of 65 -percent relative humidity and $70^{\circ} \mathrm{F}$. These values are given for the 6 -hour treatment.

Each of the dried yarns failed to regain the amount of moisture it held originally. Yarns heated at the high humidity had a lower moisture content on conditioning than those heated at the low humidity, with the exception of the acetate yarn. The moisture contents of the acetate yarns on conditioning show little effect with respect to humidity, but the effect of heat is important. For example, yarns heated at $302^{\circ} \mathrm{F}$ for 6 hours had a moisture content of only 5.3 percent on conditioning at 65 -percent relative humidity, $70^{\circ} \mathrm{F}$, as compared with the 7.3 percent moisture content of the original sample for the same conditions.

Figure 4 shows the effects of the 6-hour treatments on the affinities of the various textile materials for dyes, as measured by changes in their spectral reflectances relative to $\mathrm{MgO}$. An examination of figure 4 shows that the affinity of the dried cellulosic fibers for the dye benzopurpurine $4 B$ decreased, as indicated by the higher reflectance values. Also, the fibers heated at the high humidity for a given temperature adsorbed less of this dye than when heated at the low humidity. This is in agreement with the physical and chemical data given above.

The wool yarns, on the other hand, when degraded by heat and humidity treatments adsorbed more of the acid dyes. The wool yarns heated at the high humidity at a given temperature adsorbed more of the acid dyes than when heated at the low humidity, as indicated by the lower refiectance values. In fact, there is little difference in the reflectance at a low humidity for any of the temperatures investigated, but a high humidity even at the lowest temperature investigated, $221^{\circ} \mathrm{F}$, gave low reflectance compared with the original. This difference in affinity of the dried clothing wool yarn for dyes corresponds to the difference in the cystine content (see table 3).

Figure 5 shows the relationship between breaking strength and fluidity of the dried and conditioned textile materials. The curves for the viscose rayon, the purified cotton, and the degummed silk indicate linear relations between fluidity and breaking strength, provided the humidity is taken into consideration. Little relationship was found between breaking strength and fluidity for the raw cotton. The changes in breaking strength and fluidity are small compared with those of the viscose-rayon and the purified-cotton yarns. The sharp break upward in the curve for the raw cotton yarn does indicate that the fluidity is more sensitive to changes in the fiber than the breaking strength of the yarn.

The acetate yarn is peculiar in that no relationship was found between breaking strength and fluidity. However, elongation plotted 
against the corresponding fluidity gave an approximate linear relationship. This indicates that breaking strength is the least sensitive of the criteria used for judging degradation in the acetate yarn.

Figure 6 shows a definite relationship between the reflectance and the chemical and physical properties. The reflectances of the purified cotton and the viscose-rayon yarns change more rapidly than the fluidities in the first stages of apparent degradation. This may indi-
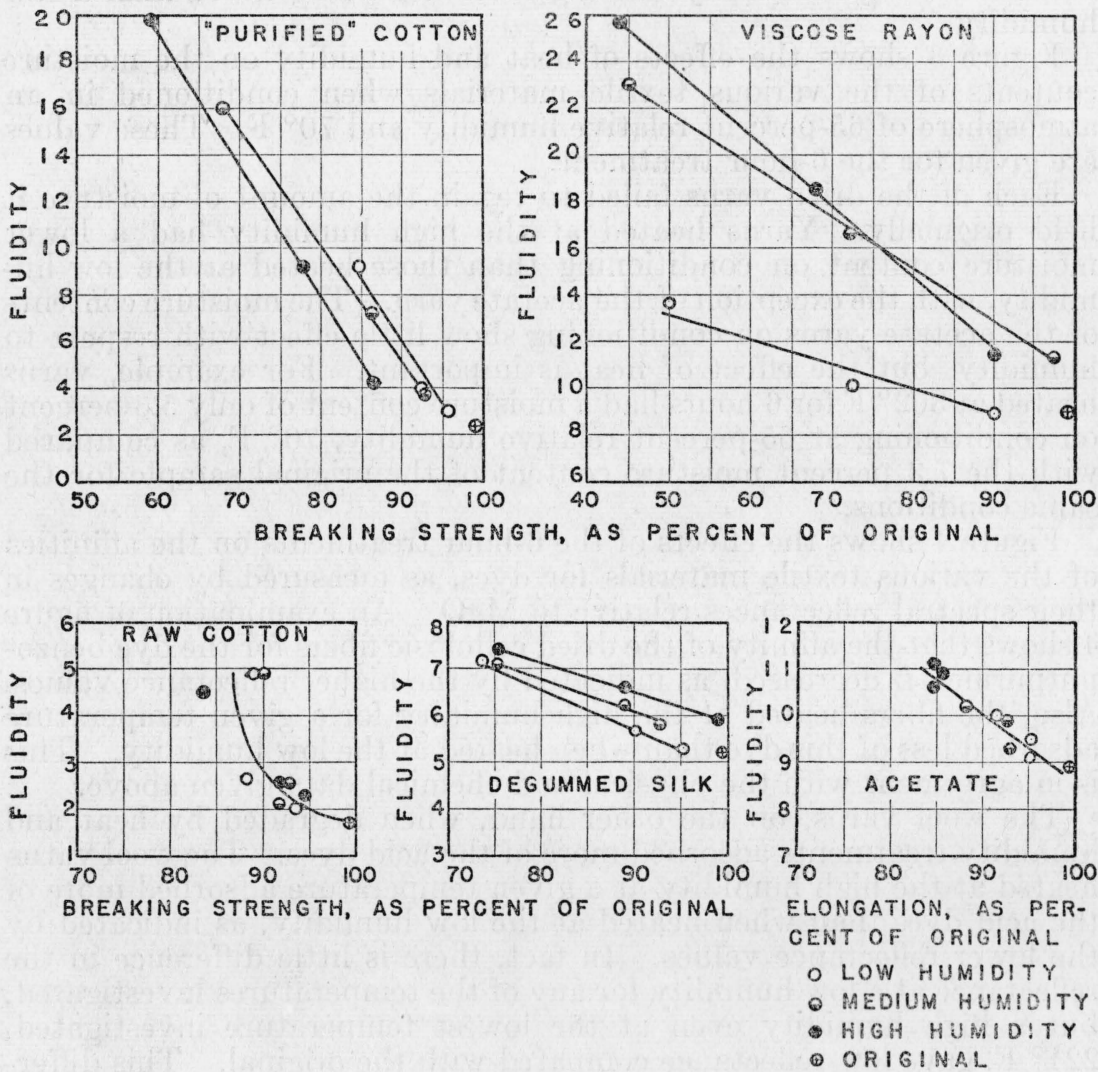

FiguRe 5.-Relationship between fluidity and breaking strength as a funciion of humidity.

cate merely that the dye test measures surface changes in the yarns, and that the fluidity measures mainly chemical changes.

The time of heating the yarns is also important. The conclusions mentioned above were drawn on the basis of the data for the 6-hour treatment. The tables give the results for short intervals of time as well as for the 6 -hour period. An examination of tables 1 to 9 shows that for the shorter periods studied, $1 / 2,3 / 4$, and 1 hour, most of the materials underwent very little decrease in breaking strength. A slightly greater effect on elongation at break is indicated. Several exceptions, however, are noted. There was some lowering in breaking strength and elongation at break of the carpet wool and acetate yarns when heated for a 2 -hour period at $221^{\circ} \mathrm{F}$ at the high humidity. The viscose- and cuprammonium-rayon yarns, which were the least 
resistant to heat of all the materials studied, showed a decrease in breaking strength and elongation if heated for only $1 / 2$ hour at $302^{\circ} \mathrm{F}$. The purified cotton yarn showed a small decrease in quality index on heating for $1 / 2$ hour at $302^{\circ} \mathrm{F}$.

There was an increase in fluidity of the viscose-rayon yarn after heating it for a short period at $257^{\circ} \mathrm{F}$ at the high humidity, and for
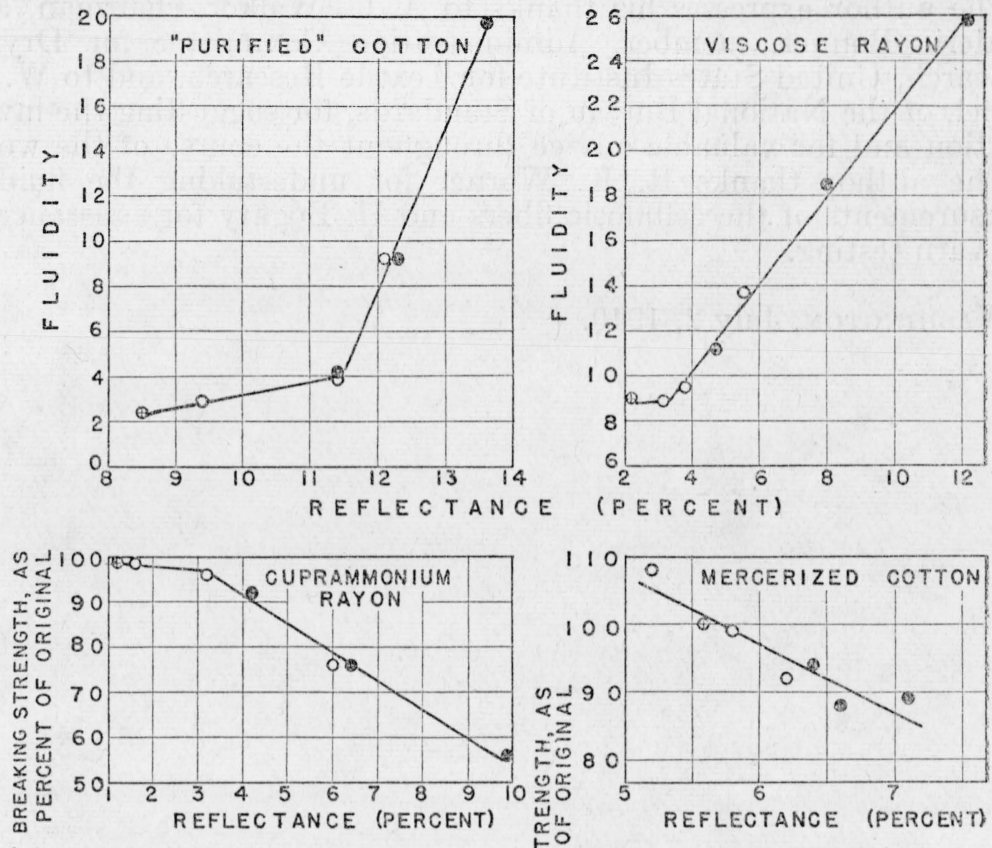

总
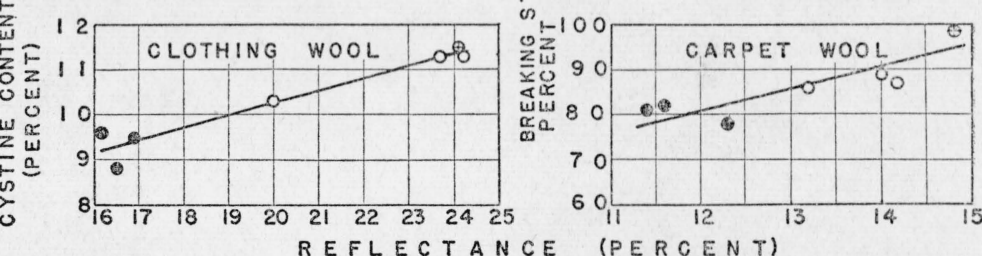

OLOW HUMIDITY

DHIGH HUMIDITY

ФORIGINAL

FIGURE 6.-Relationship between reflectance and the physical and chemical data as a function of humidity.

a short period at $302^{\circ} \mathrm{F}$ at all three humidities. The fluidity of the purified cotton yarn was approximately 4.0 rhes after $1 / 2$-hour treatment at $302^{\circ} \mathrm{F}$ at all humidities, 9.1 rhes after $1 \frac{1}{2}$ hour at $302^{\circ} \mathrm{F}$ at the high humidity, as compared with the original sample with a fluidity of 2.4 rhes.

There was an increase in reflectance of dyeings of the viscose rayon, cuprammonium rayon, and purified cotton heated for only short periods of time, indicating a decrease in affinity for the dye. For example, dyeings were prepared after heating the yarns at $302^{\circ} \mathrm{F}$ for $1 \frac{1}{2}$ hours at the low humidity and at the high humidity. The 
reflectances of the untreated yarns, yarns heated at the low humidity, and yarns heated at the high humidity, respectively, were: viscose rayon, $2.1,4.4$, and 5.7 percent; cuprammonium rayon, 1.2, 1.7, and 4.3 percent; purified cotton, $8.5,10.8$, and 10.7 percent.

The author expresses his thanks to A. C. Walker, chairman, and Frederic Bonnet, member, Administration Committee for Drying Research, United States Institute for Textile Research, and to W. D. Appel, of the National Bureau of Standards, for suggesting the investigation and for valuable advice throughout the course of the work.

The author thanks R. K. Worner for undertaking the fluidity measurements of the cellulosic fibers and $\mathrm{H}$. Bogaty for assistance in the yarn testing.

Washington, July 2, 1940. 